\title{
Relationships between Bulk Silicate Moon FeO Content and Bulk Moon Physical Properties
}

Sabrina Schwinger and Doris Breuer

German Aerospace Center (DLR), Planetary Physics, Berlin, Germany (sabrina.schwinger@dlr.de)

\section{Introduction:}

Estimates of the bulk silicate Moon (BSM) composition have been proposed based on a number of different geochemical, petrological and geophysical arguments but have yet to arrive at a general consensus.

In order to obtain further constraints on the BSM composition, we investigated the effect of the BSM $\mathrm{FeO}$ content on the physical properties of lunar mantle reservoirs and tested the consistency of different lunar interior models with the bulk Moon density and moment of inertia.

\section{Methods:}

To cover all lunar interior models that are consistent with a given BSM composition, we modeled the properties of the chemical reservoirs forming from lunar magma ocean (LMO) solidification, considered the re-distribution of these reservoirs in the lunar mantle by solid state convection, and calculated the bulk Moon density and moment of inertia of the resulting interior models, assuming varying core properties and selenotherms.

\section{Lunar Magma Ocean Crystallization:}

We modeled LMO cumulate mineralogies using a combination [1] of crystallization algorithms from the software packages alphaMELTS [2] and SPICES [3], that has been validated against recent experiments on LMO fractional crystallization [4, 5]. Thereby we assumed pure fractional crystallization of a deep LMO, that extends to the core-mantle boundary so that the LMO comprises the whole BSM. The bulk LMO composition was chosen based on the estimate of [6]. FeO/MgO ratios of the bulk LMO composition were varied $(8.0-13 \mathrm{wt} \% \mathrm{FeO})$ to investigate the effect of the $\mathrm{FeO}$ content on the densities and mineralogies of individual cumulate layers. All crystals forming in the LMO were assumed to sink and equilibrate with the liquid at the bottom of the magma ocean prior to fractionation, except for plagioclase which was assumed to float to the surface to form anorthositic crust. The average lunar crust thickness was assumed to be $40 \mathrm{~km}$ in accordance with recent GRAIL data [7]. Any excess plagioclase that formed after that final crust thickness was reached was assumed to remain in the mantle due to imperfect plagioclase floatation.

\section{Mantle Mixing and Overturn:}

As a consequence of the higher compatibility of lighter $\mathrm{Mg}$ compared to denser $\mathrm{Fe}$ in the LMO cumulate minerals, the density of the cumulate increases with progressing LMO solidification. Since the LMO solidifies from bottom to top, this results in a gravitationally unstable cumulate stratification that facilitates convective overturn, during which dense material sinks towards the core mantle boundary while lighter material migrates toward the surface. The respective changes in pressure and temperature experienced by individual cumulate layers, as well as mixing and chemical 
equilibration of different layers during overturn, can affect the mineralogy and physical properties of the lunar mantle. To investigate these effects, we calculated equilibrium mineral parageneses of different cumulate layers using Perple_X [8]. For simplicity we considered five homogeneous cumulate reservoirs (olivine-dominated, pyroxene-dominated, IBC, KREEP and crust), whose compositions were derived from the results of the LMO crystallization models by averaging the compositions of adjacent cumulate layers with similar mineralogies.

The mineralogies and densities of each reservoir were calculated as a function of depth along different selenotherms (e.g. [9]). To evaluate the effect of mixing and chemical equilibration, we also made the same calculations for different compositional mixtures of the layers.

The results of these calculations were used as input in a simple density structure model in order to investigate the effect of mantle overturn on the bulk lunar density and moment of inertia. Lunar core sizes and densities were thereby varied within the range of proposed values (e.g. [10]).

\section{Results:}

Changing the $\mathrm{FeO} / \mathrm{MgO}$ ratio of the BSM composition leads to an earlier appearance and higher abundance of Fe-rich minerals in the LMO cumulate. This results in an increased thickness of the late formed, dense ilmenite bearing cumulate (IBC) reservoir, that we defined based on its high density compared to underlying cumulate layers. As a consequence, IBC thickness correlates linearly with the assumed LMO FeO content, varying by a factor of about 4 over the assumed range of FeO contents.

Due to its high density the radial distribution of IBC material in the lunar interior has a significant effect on the BSM moment of inertia, even though its volume is comparatively small. The effect of the distribution of IBC on the BSM moment of inertia increases systematically with increasing IBC volume, which is in turn linked to the FeO content.

Depending on the chosen core models and selenotherms, realistic bulk Moon densities and BSM moments of inertia could be reached assuming $\mathrm{FeO}$ contents of $8-13.5 \mathrm{wt} \%$.

\section{Discussion:}

The range of possible FeO contents determined here is valid for a large variation of lunar interior properties and can be narrowed considerably by imposing constraints on the selenotherm, core properties or mantle structure. Seismic and selenodetic data indicate a mantle stratigraphy with a pyroxenitic upper mantle and a dunitic lower mantle [11] and suggest the presence of a dense, partially molten zone at the core mantle boundary [12], that might consist of sunken IBC material. These constraints on the interior structure of the lunar mantle indicate that BSM FeO contents of 9 $11 \mathrm{wt} \%$ are most probable. This estimate could be further limited by tighter constraints on the size and density of the lunar core, e.g. by future seismic investigations.

\section{References:}

[1] Schwinger and Breuer (2018), AGU Fall Meeting, Washington, USA.

[2] Smith and Asimow (2005), G3 6.2 .

[3] Davenport (2013), Planet. Sci Res. Disc. Report 1, 173.

[4] Rapp and Draper (2018), Meteoritics \& Planet. Sci. 53.7, 1432-1455.

[5] Charlier et al. (2018), Geochim. Cosmochim. Acta, 234, 50-69.

[6] O'Neill (1991), Geochim. Cosmochim. Acta, 55 (4), 1135-1157.

[7] Wieczorek et al. (2013) Science, 339, 671.

[8] Connolly (2005), EPSL, 236 (1-2), 524-541.

[9] Laneuville et al. (2013), JGR: Planets, 118 (7), 1435-1452.

[10] Weber et al. (2011), science, 331 (6015), 309-312.

[11] Gagnepain-Beyneix et al. (2006), Physics of the Earth and Planetary Interiors, 159 (3-4), 140-166.

[12] Matsumoto et al. (2015), GRL, 42 (18), 7351-7358. 